\title{
Estimating PageRank deviations in crawled graphs
}

\author{
Helge Holzmann ${ }^{1 \dagger}$, Avishek Anand ${ }^{2}$ and Megha Khosla $2^{*}$ (D)
}

\footnotetext{
*Correspondence: khosla@|3s.de tThe work was conducted while the author was a PhD student at L3S Research Center, Hannover, Germany.

${ }^{2}$ L3S Research Center, Leibniz University, Appelstrasse 9a, 30167 Hannover, Germany Full list of author information is available at the end of the article
}

\begin{abstract}
Most real-world graphs collected from the Web like Web graphs and social network graphs are partially discovered or crawled. This leads to inaccurate estimates of graph properties based on link analysis such as PAGERANK. In this paper we focus on studying such deviations in ordering/ranking imposed by PAGERANK over crawled graphs. We first show that deviations in rankings induced by PAGERANK are indeed possible. We measure how much a ranking, induced by PAGERANK, on an input graph could deviate from the original unseen graph. More importantly, we are interested in conceiving a measure that approximates the rank correlation among them without any knowledge of the original graph. To this extent we formulate the HAK measure that is based on computing the impact redistribution of PAGERANK according to the local graph structure. We further propose an algorithm that identifies connected subgraphs over the input graph for which the relative ordering is preserved. Finally, we perform extensive experiments on both real-world Web and social network graphs with more than $100 \mathrm{M}$ vertices and $10 \mathrm{~B}$ edges as well as synthetic graphs to showcase the utility of HAK and our High-fidelity Component Selection approach.
\end{abstract}

Keywords: PageRank, Crawls, Ranking deviations

\section{Introduction}

Most real-world graphs collected from the Web like Web graphs and social network graphs are partially discovered (crawled) or in other words their graph topology is not known in entirety ( $\mathrm{Li}$ et al. 2015), especially if not crawled for a particular purpose or subset, but extracted from existing crawls, such as Web archives. The goal of Web archive crawlers is to capture as much as possible starting from some seed set within some national domain or even broader, given the available but limited resources (Costa et al. 2017). Complicating matters further, Web archives are often not constructed in one piece but by merging partial crawls (Holzmann et al. 2016). Additional reasons for missing information (links) in Web archives include the restrictive politeness policies (i.e., robots.txt) or random timeouts of Web servers. Several studies on this topic have shown that the problem of partial discovery is indeed a common issue (Huurdeman et al. 2014), inevitably affecting the graphs extracted from such crawls as well.

As a result, important graph properties and measures used for link analysis and structural characterization like authority of vertices might be inherently flawed or exhibit deviations from their original values. This is commonly observed where users are typically agnostic to the incompleteness of the obtained graph, hoping that the input graph

c) The Author(s) 2019 Open Access This article is distributed under the terms of the Creative Commons Attribution 4.0 International License (http://creativecommons.org/licenses/by/4.0/), which permits unrestricted use, distribution, and reproduction in any medium, provided you give appropriate credit to the original author(s) and the source, provide a link to the Creative Commons license, and indicate if changes were made. 
is a reasonable representative sample of the underlying (unseen) original graph. Some of the well-known measures for computing authority of vertices or relative ordering of vertex authorities based on random walks are PAGERANK (Page et al. 1999) and its variants (Kleinberg 1999; Haveliwala 2002).

As an example, consider PAGERANK computed over the .gov Web graph that we will analyze in detail later in this work. Here, the women.nasa.gov(Women@NASA) page has a high PAGERANK value and is subsequently found within the top 300 pages. However, on a closer examination we observe that most of its PAGERANK is contributed by an in-link from the highly popular NASA homepage (nasa.gov). If for some reason this particular in-link is not crawled, e.g., due to a temporary downtime or the decision by $N A S A$ to exclude their homepage from being crawled, this would cause a large decrease in its PAGERANK and hence a severe rank deviation in the obtained crawl.

One might argue that this is an unlikely case since important pages enjoy a high priority and are therefore commonly crawled, but this might not always be the case in reality. To support our claim we performed the following experiment. We ranked pages in a graph constructed from a . de Web archive in $2012^{1}$ based on (1) inlinks and (2) PAGERANKS. The above mentioned graph considered only links that emerged in 2012 (Holzmann et al. 2017). We then checked if the top ranked pages in this crawled graph were indeed archived in that year. Our experiments show that from among the top 1000 pages, ranked according to inlinks, roughly $30 \%$ are contained in the archive. According to PAGERANK rankings, less than $20 \%$ of the top 1000 pages are contained in the archive. With this small experiment we show that high priority vertices can indeed be missed in real world crawls, which can further cause a rank deviation in the obtained crawled graph.

In this paper we focus on studying such deviations in ordering/ranking imposed by PAGERANK over crawled graphs. Vertices in our input crawls are either completely crawled (all neighbors are known) or are uncrawled (none of their outgoing neighbors are known but their incoming links from the crawled vertices are known), which we also refer to as ghost vertices. In this paper, we focus on the following 3 research questions.

- RQ I : Do crawls or partially discovered real-world graphs show a deviation in their PAGERANK orderings when compared to full network topology?

- RQ II : How can we reliably measure the extent of such ranking deviations for crawled graphs?

- RQ III : Can we identify suitable sized components in a graph for which PAGERANK computations are reliable?

We first establish empirically that real-world networks indeed show a deviation in their PAGERANK orderings when not crawled completely compared to the full graph (RQ I). We present ranking correlations (measured by Kendall's Tau) dropping down to 0.55 on Web graphs when only $50 \%$ of it is crawled. That corresponds to an average positional displacement of 66,216 ranks among the top $30 \%$ pages in the above mentioned .gov graph. We note that for most of the applications, only ranking deviations of the top pages are interesting. In addition the results from (Ng et al. 2001a) imply that vertices with small actual PAGERANKS (in the original, unperturbed graph) will have smaller deviations with respect to their original scores in the modified or crawled graph.

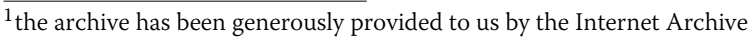


Users and applications that use rankings induced by PAGERANK as a feature for downstream ranking and learning tasks would naturally be interested in estimating such a deviation from the (crawled) input graph at hand as a measure of confidence. As an answer to RQ II, we propose a measure called HAK (an acronym of the authors' lastnames) that estimates the ranking deviation of an crawled input graph when compared to the original graph. Note that at all times we are agnostic about the original (unseen) target graph and hence, the estimates are purely based on the observed input graph. Also, our target graphs are large Web or social network graphs and hence, the computation of such a measure should be scalable. The HAK measure relies on local estimates of vertex-level attributes and can be easily computed in a distributed manner.

Our third contribution, towards RQ III, is to propose an algorithm for the selection of maximum connected subgraphs in the given crawls, for which the relative ordering of the vertices is preserved. In other words, the aim is to identify connected components from a crawl where the ordering among the vertices would have bounded deviations with respect to the unknown parent graph. This is essential for users to give them a more representative set of vertices over which measures can be more accurately computed, if their input graph suggests strong ranking deviations.

Finally, we perform extensive experiments on both real-world Web and social network graphs with more than 100 million vertices and 10 billion edges as well as synthetic graphs to discuss the utility of HAK and propose a new algorithm, which we refer to as Highfidelity Component Selection, in order to find components from a given crawl that have relatively lower ranking deviations.

Our results, apart from measuring the deviations in PAGERANKS, also shed light on the structure of the Web and the nature of Web graphs in general. We observe that Web graphs behave more (although not completely) like random graphs under certain crawling strategies.

We remark that the main results corresponding to RQ I and RQ II appeared in the preliminary version of the paper(Holzmann et al. 2018). The present work in addition investigates RQ II towards which new algorithm is proposed and evaluated. In addition, we provide a more detailed analysis and discussion on the results of the other two research questions.

\section{Related work}

$\mathrm{Ng}$ et al. (2001a) analyzed the conditions under which eigenvector methods like PAGERANK and HITS can provide reliable rankings under perturbations to the linkage patterns for a given collection. In particular for PAGERANK they showed that if the perturbed or modified web pages (a web page is perturbed/modified for example when the links from the page are removed or are not followed) did not have a high overall PAGERANK scores in the original graph, then the new PAGERANK scores will not be far from the original. Though they bound the variational distance between the PAGERANK distributions of the original and the modified graph in terms of the original PAGERANK scores of the modified pages and the teleportation probability, they provide no insight of how to estimate such a measure when only the modified graph or the crawl is given. We, on the other hand, provide a measure to estimate ranking deviations of vertices in the given graph with respect to their orderings in the original unmodified graph. $\mathrm{Ng}$ et al. (2001b) uses the insights from (Ng et al. 2001a) to propose two new algorithms: Randomized HITS, which merges 
the hubs-and-authorities notion from HITS with a stabilizing "reset" (teleportation to a random vertex) mechanism from PageRank; and Subspace HITS, which provides a principled way of combining multiple eigenvectors from HITS to yield aggregate authority scores.

Bianchini et al. (2005) analysed the stability among other properties of PageRank. In essence, they show that overall change of PageRank the whole Web is proportional to the sum of PageRank of the perturbed pages, i.e, PageRank is robust with respect to changes taking place in small communities of the Web. Their theoretical analysis provide a stronger bound as compared to (Ng et al. 2001a) on change in PageRank in presence of perturbations. Similar to (Ng et al. 2001a) their result gives no insight on how to measure the discrepancy if only the modified graph is known.

Ghoshal and Barabási (2011) predicts analytically the presence of a small number of super-stable nodes in scale-free networks whose ranking given by PageRank is exceptionally stable under degree-preserving perturbations. The number of these super stable nodes is typically very small, for example with the numbers reported (Ghoshal and Barabási 2011) range from 1 to 4 . We, on the other hand, compute ranking deviations exhibited by a larger percentage of the top ranking nodes including the not so stable (towards perturbations) nodes. Moreover, the more general crawling strategies that we consider in this work may not always be degree preserving as the case studied in the above work.

Boldi et al. (2004) were the first to show the paradoxical effects of PAGERANK computation on Web graphs. They discuss a measure of effectiveness for crawl strategies based on whether the graph obtained after a partial visit is in some sense representative of the underlying Web graph for the PAGERANK computation. Similar to our setting, they study how rapidly the computation of PAGERANK over the visited subgraph yields relative ranks (measured by Kendall's Tau) that agree with the ones the vertices have in the full graph. In a later work (Boldi et al. 2006), they show a connection between graph fibrations and Markov chains with restart, a particular kind of Markov chains that include the PAGERANK one as a special case. In (Vattani et al. 2011) the authors operate on a given subset of vertices and consider the general problem of maintaining multi-scale graph structures by preserving a distance metric based on PAGERANK among all pairs of sampled vertices. Other authors investigated this problem before as well, however, none of them focused on random walk algorithms, such as the widely used PAGERANK, neither explored the effect of missing nodes in real-world Web graphs (Wang et al. 2012; Smith and Moody 2013; Smith et al. 2017).

The other area of related work comprises of graph sampling approaches which can be broadly classified into two categories: traversal based methods (Leskovec and Faloutsos 2006; Wang et al. 2010; Maiya and Berger-Wolf 2011) and random walk based methods (Gjoka et al. 2011; Li et al. 2015; Hübler et al. 2008). Graph-traversal based methods employ breadth-first search (BFS) or the depth-first search (DFS) algorithm to sample vertices and are typically shown to exhibit bias towards high-degree vertices (Wang et al. 2010). Maiya and Berger-Wolf (2011) compare various traversal based algorithms and define representativeness of a sample while proposing how to guide the sampling process towards inclusion of desired properties. On the other hand, the random walk based methods are popular for graph sampling because they can produce unbiased samples or generate samples with a known bias (Zhou et al. 2016; Gjoka et al. 2011; Li et al. 2015; 
Hübler et al. 2008). Other works have directly tried to estimate graph properties such as degree distribution estimation (Gjoka et al. 2011), clustering coefficient estimation (Hardiman and Katzir 2013), size estimation (Katzir et al. 2011), and average degree estimation (Dasgupta et al. 2014). One of the popular sampling algorithms used for Web graphs is the Forest Fire algorithm by Leskovec and Faloutsos (2006), a generative graph model, in which new edges are added via an iterative "forest fire" burning process where it is shown to produce graphs exhibiting a network community profile plot similar to many real-world graphs. We use this approach in generating synthetic real-world graphs.

However, most of these works, with the exception of (Li et al. 2015), assume a known graph topology. Our work focuses on the unknown graph topology, an arguably more general and useful scenario in Web graphs and social networks gathered by crawlers. In addition, with our component selection approach we optimize for a more reliable PAGERANK ordering instead of other properties like degree distributions or clustering coefficients as considered in earlier works.

\section{Preliminaries and problem}

PageRank. As originally conceived, PAgERANK ranks vertices of a directed graph $\mathcal{G}=$ $(V, E)$ where $V$ and $E$ are the vertices and edges respectively, based on the topological structure of the graph using random walks (Page et al. 1999). The problem we are addressing in this paper is attributed to this random walk model behind PAGERANK, representing the authority or importance of a vertex.

For some fixed probability $\alpha$, a surfer at vertex $v \in V$ jumps to a random vertex with probability $\alpha$ and goes to a linked vertex with probability $1-\alpha$. The authority of a vertex $v$ is the expected sum of the importance of all the vertices $u$ that link to $v$. Consequently, a vertex receives a high PAGERANK value and is ranked at the top by ordering the webpages by importance when it is either connected by many incoming edges or reachable from another important page.

We first define the notions of target graph, crawl and ghost vertices in the context of partial discovery/sampling in graphs:

Definition 1 (Target graph) The subset of vertices (with the induced edges) of a larger graph (e.g., the Web) that is theoretically reachable by a crawler given its seeds, e.g., a domain, a top-level domain, or all webpages that belong to a certain topic in case offocused crawlers. This graph would be available if every link was followed and every page captured by the crawler, illustrated by the target in Fig. 1.

Definition 2 (Crawled graph or Crawl) The (partial) graph derived from the set of webpages that have actually been visited by the crawler, discovered/linked yet uncrawled pages are not included. This subset of the target graph is illustrated by the crawl in Fig. 1.

Definition 3 (Ghost vertex) Although a hyperlink on a crawled page points to another page that belongs to the target graph, there is a chance the crawler never crawled that page, yet it has discovered its existence as it was pointed from one of the crawled pages. Such a page or vertex, which is never crawled (or its outgoing neighbors are not known) but its existence and incoming edges from other crawled page/pages are known, is referred to as ghost vertex, shown by the gray vertices outside the crawl in Fig. 1. 


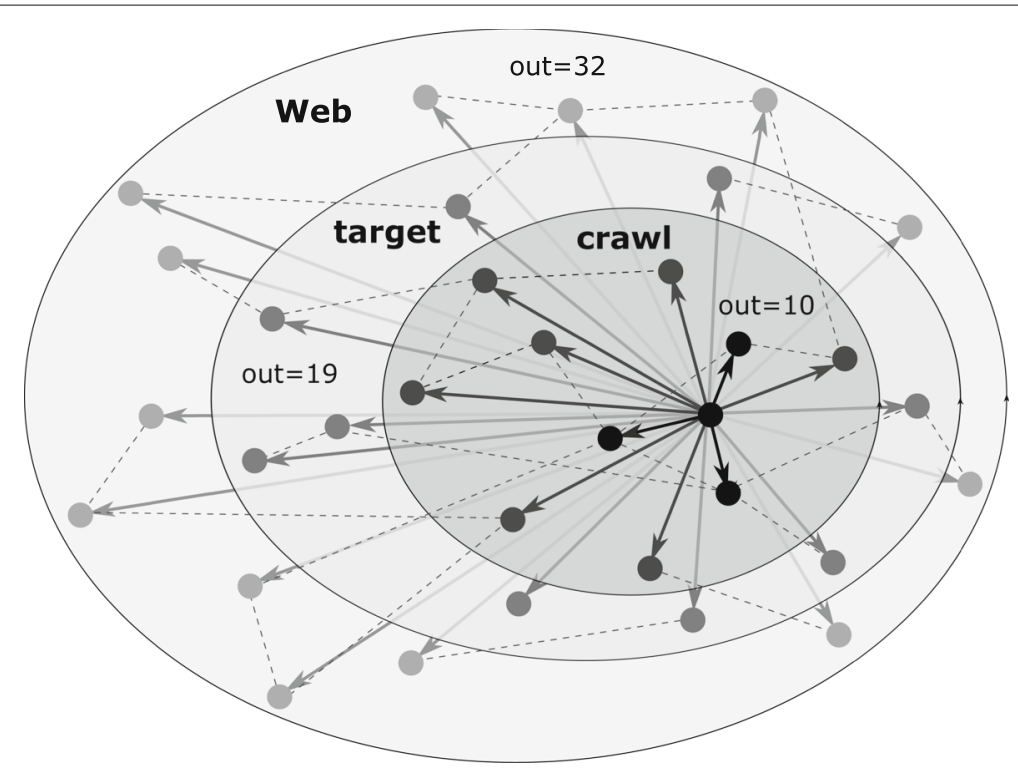

Fig. 1 Neighborhood of a webpage in different subgraphs of the Web

Ranking Deviations. The deviation among two rankings induced by PAGERANK is a global objective, independent of a specific query. Hence, local or relevance-based measures such as nDCG are not applicable here. The most common metrics to quantify rank correlation are Spearman's Rho and Kendall's Tau, which are both similar as they are special cases of a more general correlation coefficient and measure relative displacements.

In this work, we use Kendall's Tau (Kendall 1938), ranging from $[-1,1]$, with 1 corresponding to a perfect rank correlation, 0 corresponding to no correlation and -1 to a perfect inverse correlation, to compare the correlation/deviation of rankings computed on the vertices of a crawl $\mathcal{G}_{C}$ with respect to that of the target graph $\mathcal{G}_{T}$.

In Fig. 2 we provide a few examples of possible graph structures, where partial knowledge of the graph may affect the ranking returned by the PAGERANK values. We remark that in the next sections, we will also provide empirical evidence, supporting the fact that there exists ranking deviations in crawls of some real-world graphs. In the first subfigure (a), we show the positive case of a DAG where the partial knowledge of the graph will not cause any ranking deviations. As only the topmost vertices shown here receive significantly more links than the others, these are also the most important vertices. It is easy to see here that generating a crawl from this structure by removing some vertices will not cause any significant changes in the ranking orderings of the crawl. In the next subfigure (b), a backlink has been introduced (left) that feeds back the importance of a top most page to a previously unimportant page and its successors. This importance gets propagated through the cycle which has been created due to the inserted backlink. In the next subfigure (c), we illustrate the case of a crawl in which vertices are removed uniformly at random. The changes here are that primarily unimportant vertices are removed, which would still not cause much deviations in the ranking orderings. Finally, if we remove any vertex from the cycle as shown in subfigure (d), its succeeding vertices drastically lose in importance and hence, the ranking among the pages in the crawl changes noticeably. 


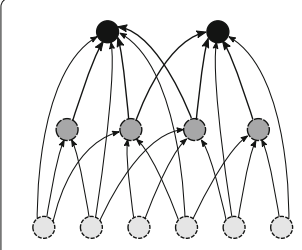

(a)

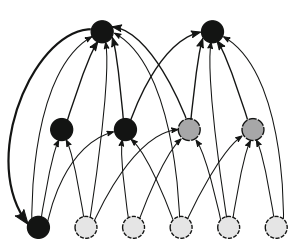

(b)

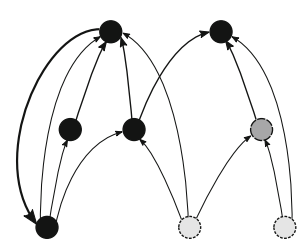

(c)

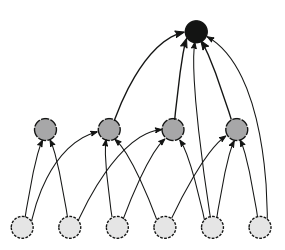

(d)

Fig. 2 Example graphs: Darker vertices have a higher importance (cp. "Preliminaries and problem" section)

\section{The HAK measure}

With our measure, we estimate quantitatively how reliable a crawl is with respect to the relative ordering of the PAGERANK values on its vertices compared to the corresponding target graph. To this end, we first try to estimate the size of the target graph: Given the crawled vertex set and the distinct hyperlinks on the corresponding webpages, some of which are pointing to an uncrawled page (ghost vertex), how big is the target graph or a subgraph that would potentially impact or contribute to the PAGERANK values of the vertices in the crawl? We show that for simple crawling strategies where it can be assumed that each vertex is part of the crawl independently from all other vertices with some sampling probability $p_{s}$, the size of the target graph can be estimated in terms of a very simple property of the crawled vertices, namely, the fraction of its crawled neighbors, referred to as fidelity. Secondly, we try to estimate the impact exerted by the vertices in the target graph on the crawled vertices, which we in turn use to estimate the number of discordant pairs in the crawl. We now describe in detail the construction of the HAK measure. Let $C=\left(V_{C}, E_{C}\right)$ denote the crawled graph and let $n$ be the number of vertices in this graph. The main steps in our computation are as follows:

1 Estimate the size of the target graph by using connectivity properties of the crawl.

Let $T=\left(V_{T}, E_{T}\right)$ represent the target graph.

2 Estimate the impact (as functions of PAGERANK) of the vertices in $V_{C}$.

3 Assume that the vertices in $V_{T}$ exert similar impacts on other vertices.

4 Estimate the number of discordant pairs due to impacts exerted by vertices in $V_{T}-V_{C}$ on vertices in $V_{C}$.

\section{Estimating the target graph}

Let $\mathcal{N}$ denote the number of vertices in the target graph. In this section we will estimate the value of $\mathcal{N}$ assuming that the crawl is constructed by sampling vertices from the target graph independently and uniformly at random with some probability $p_{s}$. Note that if $p_{s}$ is known, one can easily estimate $\mathcal{N}$ as $\frac{n}{p_{s}}$. We therefore first estimate $p_{s}$ from the connectivity properties of the crawl and the target graph, for which we need the following definition:

For any vertex $v \in V_{T}$, we define fidelity $(\gamma(v))$ of $v$ as the ratio of its immediate neighbors in $V_{C}$ to its total out-degree (number of distinct hyperlinks on a webpage pointing to vertex in $V_{T}$ ). Let $d_{c}(v)$ count the number of vertices $v^{\prime} \in V_{C}$ reachable from $v$ in one step (hop) and $d(v)$ denotes the total out-degree of $v$.

Definition 4 (Fidelity) The fidelity of a vertex $v \in T, \gamma(v)$, is given by $\gamma(v)=\frac{d_{c}(v)}{d(v)}$ and the average fidelity of all vertices in $C$ is $\gamma(C)=\frac{\sum_{v \in V_{C}} \gamma(v)}{n}$. 
We will now show that $\mathbb{E}(\gamma(v))=p_{s}(1-\mathbb{P}(d(v)=0))$. In essence we would like to estimate the unknown sampling probability that is used to generate the crawl using empirically computable fidelity of the observed crawl.

Proposition 1 Let for some $0<p_{s}<1$, each vertex in the target graph is sampled independently and uniformly at random with probability $p_{s}$. For any $v \in T, \mathbb{E}(\gamma(v))=$ $p_{s}(1-\mathbb{P}(d(v)=0))$.

Proof The probability that a vertex has fidelity $\ell / k$ is given by

$$
\mathbb{P}\left(\gamma(v)=\frac{\ell}{k}\right)=\mathbb{P}\left(d_{c}(v)=\ell \mid d(v)=k\right) \cdot \mathbb{P}(d(v)=k)=\left(\begin{array}{l}
k \\
\ell
\end{array}\right) p_{s}^{\ell}\left(1-p_{s}\right)^{k-\ell} \mathbb{P}(d(v)=k) .
$$

The expected value of fidelity of $T$ can now be computed as

$$
\begin{aligned}
\mathbb{E}(\gamma(v)) & =\sum_{k \geq 1} \sum_{\ell \leq k} \frac{\ell}{k}\left(\begin{array}{l}
k \\
\ell
\end{array}\right) p_{s}^{\ell}\left(1-p_{s}\right)^{k-\ell} \mathbb{P}(d(v)=k) \\
& =p_{s} \sum_{k \geq 1} \mathbb{P}(d(v)=k) \sum_{\ell=1}^{k}\left(\begin{array}{l}
k-1 \\
\ell-1
\end{array}\right) p_{s}{ }^{\ell-1}\left(1-p_{s}\right)^{k-\ell}=p_{s}(1-\mathbb{P}(d(v)=0)) .
\end{aligned}
$$

With $p_{s}$ as the sampling probability, $p_{s} \mathcal{N}$ gives us the expected number of vertices in the crawl. Using Proposition 1 we obtain

$$
\mathcal{N}=\frac{\mathbb{E}\left(\left|V_{C}\right|\right)}{\mathbb{E}(\gamma(v))}(1-\mathbb{P}(d(v)=0)) .
$$

We note that for Web graphs $\mathbb{P}(d(v)=0)$ is the probability that a webpage has no links to other webpages, i.e., there exists a page with pure text and no links. Such a scenario is extremely rare on the Web. Moreover, for synthetic graphs generated using $G_{n, p}$ (s. "Synthetic graphs" section) one can show that $\mathbb{P}(d(v)=0)=e^{-O(n p)}$, which goes to zero for $n \rightarrow \infty$ and constant $p$. Note that as we do not have complete information about the target graph we cannot compute $\gamma(v)$ for all vertices in $V_{T}$. We instead approximate it by the observed average fidelity of the crawl, $\gamma(C)$. This amounts to assuming that the degree distribution of target graph with respect to number of pointing inside the crawl and outside is homogeneous for all vertices in the target graph. Hence, using the observed average $\gamma(C)$ and the observed size of the crawl, i.e., $n$, ignoring the multiplicative factor of $1-\mathbb{P}(d(v)=0)($ as $\mathbb{P}(d(v)=0)=o(1)$ for all practical purposes), we can approximate $\mathcal{N}$ as $\frac{n}{\gamma(C)}$.

\section{PageRank and impacts}

In this section we present a heuristic to measure the PAGERANK contribution of a vertex to its neigbors in the crawl, which we then use to estimate the contribution of ghost vertices to their neigbors in the crawl, as if the PAGERANK algorithm ran on the whole target graph. In the following, we first define a measure that we will extrapolate for the ghost vertices in the next subsection.

For any vertex $v$ in $C, \pi(v)$ denotes its computed PAGERANK in the crawl. Let $d(v)$ denote the out-degree of $v$ and $N(v)$ denote the neighbors of $v$ reachable from $v$ in one step. Note that PAGERANK of any vertex $u$ can be considered as $\pi(u)=\sum_{v: u \in N(v)} \frac{\pi(v)}{d(v)}$ 
(Haveliwala 1999). We then define the impact of a vertex $v \in C$ on a neighbor $u \in N(v)$ as

$$
\operatorname{Im}(v, u)=\frac{\pi(v) / d(v)}{\pi(u)} .
$$

The impact on any vertex $u \in V$ is $\frac{1}{\pi(u)} \sum_{v: u \in N(v)} \frac{\pi(v)}{d(v)}$, which is always 1 . This implies that an extra impact of $x$ on a vertex will increase its PAGERANK by $x$ times the current PAGERANK. The total impact of a vertex $v, \operatorname{Im}(v)$ is then defined as

$$
\operatorname{Im}(v)=\sum_{u \in N(v)} \operatorname{Im}(v, u)=\sum_{u \in N(v)} \frac{\pi(v) / d(v)}{\pi(u)}=\frac{1}{d(v)} \sum_{u \in N(v)} \frac{\pi(v)}{\pi(u)} .
$$

We denote the average of impacts of vertices in $C$ by $\operatorname{Im}(C)$, i.e. $\operatorname{Im}(C)=\frac{\sum_{v \in C} \operatorname{Im}(v)}{n}$.

In the next subsection we will compute the impact of ghost vertices on the vertices in the crawl assuming that each ghost vertex exerts on average an impact of $\operatorname{Im}(C)$ on its neighbor.

\section{Estimating the impact of ghost vertices}

We next compute the impact that could have been exerted by the ghost vertices on the crawled vertices. For this we assume that the impact of each vertex in $T$ is $\operatorname{Im}(C)$, which gives us the total impact, $\mathcal{I}$, exerted by ghost vertices as

$$
\mathcal{I}=|T-C| \cdot \operatorname{Im}(C)=n\left(\frac{1}{\gamma(C)}-1\right) \cdot \operatorname{Im}(C) .
$$

To understand the intution behind this extrapolation of impacts, consider the extreme case, i.e., when $\operatorname{Im}(C)$ is close to 0 . This can happen when for vertices in the crawl $\gamma(v)$ is very small, i.e., vertices in the crawl have relatively high number of ghost neighbors. Note that we estimate the ground truth, i.e., the PAGERANK of vertices in the target graph with respect to (personalized to) the crawl. This would mean that at each step of the random walk, there is a higher probability of coming back to a vertex in the crawl (as compared to the probability of going back to some random ghost vertex). This intuitively means that ghost vertices (in this setting where we use crawl as the seed) cannot generate impact much more than the impact initially generated by the crawl. Similarly $\operatorname{Im}(C)=1$ implies that none of the vertices in the crawl has a ghost neighbor, i.e., $\gamma(C)=1$, and therefore, the total impact of the ghost vertices will be 0 . In terms of computing the PAGERANK in the target graph, this implies that the set of ghost vertices is unreachable from the crawl, and hence, will have no effect on the PAGERANK of vertices in the crawl as the crawl acts as the seed set.

Some of this extra impact (generated due to ghost vertices) will be acquired by some or all of the vertices in $C$, changing their PAGERANK values accordingly. This is reflected by the pairs of each two crawled vertices $(v, u) \in C \times C$ for which their order in the ranking flip, i.e., discordant pairs, or is preserved, i.e., concordant pairs. Such flips or discordant and concordant pairs of vertices are the main ingredients of Kendall's Tau and also estimated in HAK.

Now based on the graph structure this extra impact can be divided among the vertices of the crawl in several ways. For example, it can happen that the vertex with the lowest PAGERANK receives the total impact, increasing its PAGERANK by a large factor. In this case the number of discordant pairs is upper bounded by $n-1$. Moreover, we know from (Ng et al. 2001a) that vertices with low original PAGERANK scores will also 
have low PAGERANKS in the modified graph. Therefore, the effect of the loss of information is observed mostly on the PAGERANKS of the nodes higher in the original ranking. We checked experimentally several variants for impact distributions and the best variant, which is affirmative with our tests on real-world and synthetic graphs, is to distribute the total impact $\mathcal{I}$ equally among $\mathcal{I}$ vertices. If $\mathcal{I}$ is the number of impacted vertices in $T$, then the expected number of impacted vertices that belong to the crawled set will be

$$
I=\mathcal{I} \cdot \gamma(C) .
$$

In the worst case, each of these impacted vertices will result in forming a discordant pair with each of the unaffected vertex, we obtain the number of discordant pairs as

$$
D=(n-I) \cdot I .
$$

We recall that with our measure we aim at estimating the ranking deviation between the crawl and target graphs by means of Kendall's Tau rank correlation coefficient, which is based on concordant and discordant pairs among the rankings. As an example, if a pair of vertices from the crawled graph, $\left(v_{i}, v_{j}\right)$, have the same relative ranks in both the ranking measures ( i.e., when measured using only crawl and when measured using the target graph), then such a pair is called a concordant pair. One the other hand, if $v_{i}$ is ranked higher than $v_{j}$ in one ranking whereas the other way around in the other ranking, then it constitutes a discordant pair. Now the total number of vertex pairs in the crawled graph is $n(n-1) / 2$. We already computed an estimate of discordant pairs in (1).

We therefore compute HAK measure as follows:

$$
H A K=\frac{\# \text { concordant pairs - \#discordant pairs }}{\# \text { total pairs }}=\frac{\frac{n(n-1)}{2}-D-D}{\frac{n(n-1)}{2}}=1-4 \cdot \frac{D}{n(n-1)} .
$$

In the next section we answer RQ III by proposing an approach for finding components of the crawl which exhibit a good rank correlation with respect to the target graph.

Time Complexity. The theoretical time complexity of HAK computation is $O(m)$ where $m$ in the number of edges in the crawl. Note that the first step in order to compute HAK is the computation of fidelity of a vertex $v$ requires looking at each of its outgoing neighbors. Therefore, the computation of average fidelity of the crawl requires $O(m)$ time. The next step is to compute PageRank of all vertices in the crawl which takes $O(m)$ time. Computation of impact of crawl is again $O(m)$ as for each vertex one has to compute impacts by incorporating the PageRank of each of its outgoing neighbors. The rest of the HAK computation then requires $O(1)$ time.

\section{High-fidelity component selection}

So far, we have concerned ourselves in computing a local measure that estimates deviation of ranks induced by PAGERANK, i.e., it serves as a confidence measure for PAGERANK computation over a crawl with respect to its target graph. In this section, we propose an algorithm to identify small (connected) subgraphs from the input graph/crawl that potentially have lower rank deviations.

Ideally in such an approach, we should optimize for a high Kendall's Tau, indicating low deviation between the crawl and the target graph. However, in the absence of the target graph this is not possible. As a proxy, we should then care about identifying subgraphs 


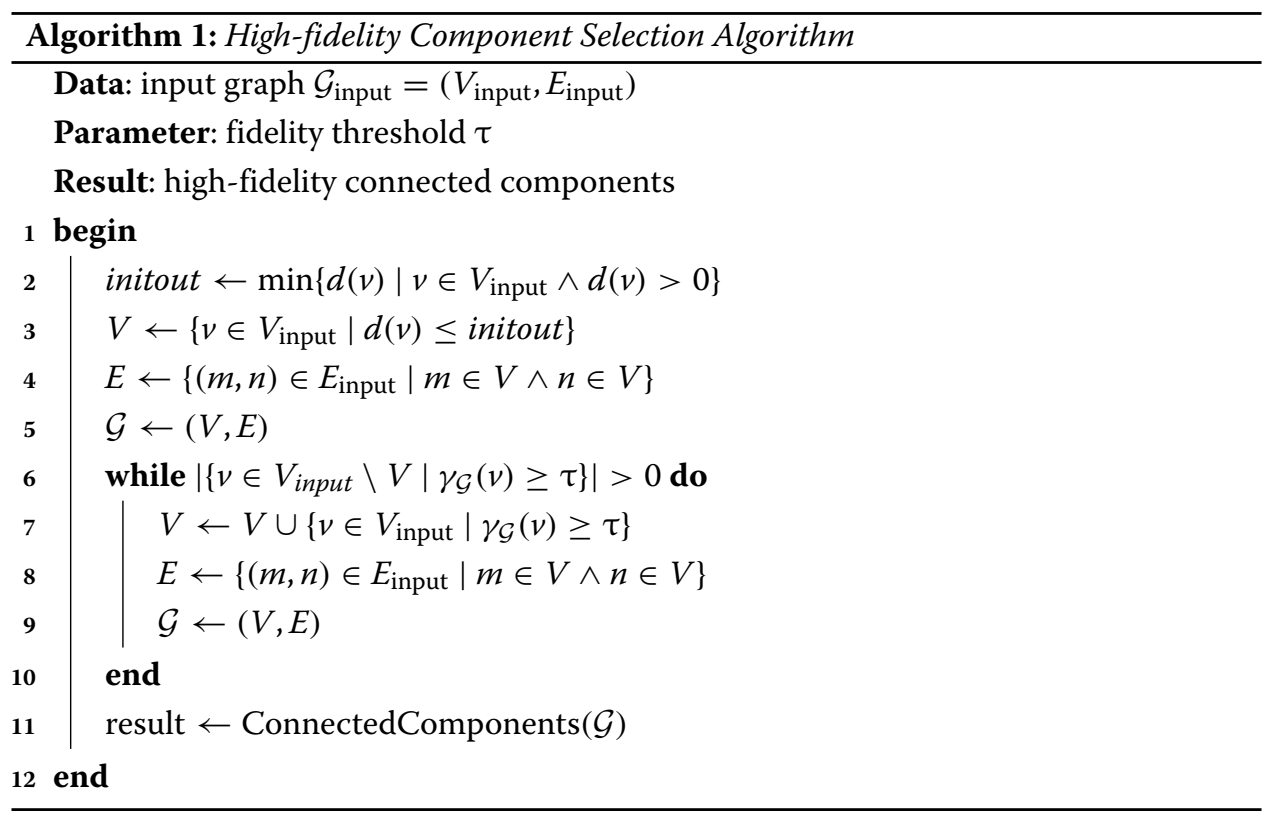

that optimize for our HAK measure, which estimates the Kendall's Tau. Unfortunately, any routine to optimize for HAK would induce an expensive global computation per candidate subgraph to compute the average impact in the graph (cp. "The HAK measure" section). Especially for Web graphs with millions of vertices, computing HAK on the refined subgraphs in each iteration is computationally infeasible. In our algorithm, we rather optimize for the most important ingredient of the HAK measure, namely fidelity of the given crawl, which we also use to estimate the target graph (cp. "The HAK measure" section). Intuitively, high fidelity in a graph translates to fewer vertices potentially losing impact in PAgERANK to ghost vertices, which in turn reduces their effect on the crawled vertices. This reduces the number of affected vertices as well as discordant pairs. In essence, the fidelity criterion can be set by the system designer based on the intended confidence level for the output graphs. We now state the formal definition of our problem of finding high-fidelity components.

Definition 5 (High-fidelity Component Selection) Given a crawl or input graph $\mathcal{G}$, we are interested in selecting a set of maximal connected subgraphs of high fidelity given a fidelity threshold of $\tau$.

Selecting such components in a graph with high fidelity turns out to be NP-hard since it is a generalization of the all-neighbors knapsack problem (equivalent when $\tau=1.0$ ) as shown by Borradaile et al. (2012). To this extent, we propose a heuristic algorithm (Algorithm 1), which intends to select the largest subgraph (possibly consisting of multiple connected components) that satisfies the fidelity criterion, i.e., the fidelity $\gamma$ for all vertices in the graph is greater than or equal to $\tau$, before splitting it into its connected components. In our algorithm described below, $\gamma_{\mathcal{G}}(v)$ refers to the fidelity of $v$ with respect to crawl graph $\mathcal{G}$ (cp. "The HAK measure" section). If $v$ is not part of this graph, $\gamma_{\mathcal{G}}(v)$ calculates the fidelity of $v$ if it was part of $\mathcal{G}$, including its outgoing edges to other vertices in $\mathcal{G}$. 
We found that the naive pruning approach, which sequentially removes vertices under the threshold, returns very small components, even for smaller values of $\tau$. Our algorithm, instead, first chooses from the input vertex set $V_{\text {input }}$ a subset $V$ that contains all vertices with either 0 out-edges (fidelity is automatically 1 , hence $\gamma(v)>\tau$ ) or the minimum non-zero out-degree in the input graph (initout). We then iteratively extend $V$ by adding vertices for which the fidelity constraint is satisfied with respect to the current graph $\mathcal{G}\left(\gamma_{\mathcal{G}}(v) \geq \tau\right)$, considering that all vertices in $V_{\text {input }}$ but not in yet $V$ are ghost vertices. The resulting subgraph is the induced subgraph on the final vertex set $V$. Finally, we return all connected components in the resulting subgraph. The algorithm can be easily implemented in a distributed manner and in practice, as we will see in the experiments, the connected components in the resulting graph actually exhibit very low variance rank deviations with respect to the ranking of the target graph.

\section{Experiments}

To validate our research questions enumerated in "Introduction" section we consider a host of large real-world graphs as well as synthetic graphs of different structures and carefully consider crawling strategies over them. In what follows we first describe our setup and rationale for our evaluation, before we discuss the results of HAK and our High-fidelity Component Selection approach.

\section{Experimental setup}

In order to evaluate HAK one require the availability of crawls as well as the target graphs that these crawls were derived from. This is necessary in order to compute how the rankings on both graphs differ and to evaluate the performance of HAK to estimate this deviation. In reality, neither obtaining the target graph is possible nor the actual crawl policy can be determined accurately. To this extent, we consider very large (as full as possible) real-world graphs under the assumption that those graphs are fully discovered ("Real-world graphs" section). We additionally simulate alternative topological structures by generating synthetic graphs ("Synthetic graphs" section). We then simulate crawls on these graphs using different crawling strategies ("Seed selection and crawling" section). For all graph and crawl combinations we ran PAGERANK on both graphs (crawl and target graphs) and compared the rankings using Kendall's Tau to evaluate HAK ("Evaluation strategy" section).

\section{Real-world graphs}

The experiments on real-world graphs were run on a computer cluster using Apache Spark and its graph processing framework GraphX (Xin et al. 2013). Loading the graphs locally on a single server was impossible with our available infrastructure because of their sizes of up to more than 100M vertices and 10B edges. As discussed earlier, we obtained multiple large real-world graphs that themselves had missing information and considered them as target graphs by discarding edges that connect to ghost vertices. The following graphs were analyzed and are summarized in Table 1:

- GOV : This graph is based on crawled webpages provided by the Internet Archive (The Internet Archive 1996). It was extracted from the latest captures of all their archived webpages under the .gov top-level domain (TLD) from 2005 to 2013. 
Table 1 Statistics on the studied real-world graphs, see "Seed selection and crawling" and "Real-world graphs" sections for details (\#V: original number of vertices, \#E: original number of edges, \#V $V_{\text {target: }}$ target number of vertices, \#E $E_{\text {target }}$ : target number of edges)

\begin{tabular}{lllll}
\hline & GOV & DE & UK & Friendster \\
\hline$\# V$ & $301,128,778$ & $247,641,473$ & $39,454,746$ & $68,349,466$ \\
$\# V_{\text {target }}$ & $5,418,054$ & $133,895,590$ & $38,838,959$ & $61,100,375$ \\
$\# E$ & $2,111,229,433$ & $14,795,732,782$ & $936,364,282$ & $2,586,147,869$ \\
$\# E_{\text {target }}$ & $180,657,788$ & $10,085,242,536$ & $928,939,162$ & $2,575,600,737$ \\
\hline
\end{tabular}

- DE : Like GOV, this . de TLD graph was also extracted from webpages archived by the Internet Archive, crawled in 2012 and generously provided to us in the project Alexandria ${ }^{2}$.

- UK : This . uk TLD crawl from 2005 is publicly available, already in the form of a graph without corresponding webpages (Boldi and Vigna 2004; Boldi et al. 2011).

- Friendster : Unlike the previous Web graphs, this is a publicly available social network, extracted from an extensive crawl of the former online platform Friendster.com in June 2011 (Archiveteam 2011).

\section{Synthetic graphs}

In order to investigate ranking deviations caused by different crawling strategies on different graph topologies, we ran a more comprehensive set of experiments on smaller, synthetically generated target graphs. This allowed for more extensive experimentation as the experiments could run locally on a single server, using the NetworkX graph analysis framework (Hagberg et al. 2008). All synthetic graphs that we studied in this work (cp. Table 2) were generated with 10,000 vertices to be reasonably sized for a thorough analysis.

The graphs were constructed using well-known graph generators, except for FFBacklinks, which is an extension by us to the ForestFire model. Although Forest Fire graphs include cycles, the model never generates backlinks from the early created vertices, which are more likely to receive many in-links over time, to newer ones. However, these links are common on the Web, where already prominent pages add links to less important ones, having a strong impact on the value propagation in PAGERANK (s. "Preliminaries and problem" section). In this graph, we added such edges between $0.05 \%$ of all pairs of an old and young vertex.

\section{Seed selection and crawling}

Crawling can be considered a special case of network sampling from a more practical point of view, where subsequent vertices can only be chosen from already discovered ones or seeds. Maiya and Berger-Wolf (2011) define this type of sampling as link-trace sampling

Table 2 Synthetic graphs (all have 10,000 vertices)

\begin{tabular}{lll}
\hline Graph generator & \#Edges & Parameters \\
\hline $\boldsymbol{G}_{\boldsymbol{n}, \boldsymbol{p}}$ (Erdós and Rényi 1959; Gilbert 1959) & 299,722 & $p \approx 0.0003$ (based on \#E in Table 1) \\
ScaleFree(Bollobás et al. 2003) & 21,732 & $\alpha=0.41, \beta=0.54, \gamma=0.05$ (default) \\
ForestFire(Leskovec et al. 2007) & 87,060 & $p_{f}=0.37, p_{b}=0.32$ (most realistic (Leskovec et al. 2007)) \\
FFBacklinks & 96,262 & $p_{f}=0.37, p_{b}=0.32, p_{\text {backlink }}=0.0005$ \\
\hline
\end{tabular}

\footnotetext{
${ }^{2}$ http://alexandria-project.eu
} 
and give a nice overview of available models for this behavior. Naturally, such approaches commonly exhibit BFS-like (Breadth-First Search) growth, but feature different strategies to prioritize or select the next vertices to be crawled. These variations determine the probability of a vertex to be part of the final sample.

How we model crawls. Although most crawlers employ BFS-like traversals, there are practical constraints like random timeouts and crawl restrictions on websites that make it hard to model crawls perfectly. Therefore, we focus on the most impartial strategy, which is vanilla BFS, but explicitely produce partial crawls by dropping randomly $x \%$ of the vertices of the input graph (where $x \in\{10,20,30,40,50\})$. We refer to this percentage as the block fraction and the remainder as desired fraction.

Statistics about the target graphs ( $V_{\text {target }}$ and $\left.E_{\text {target }}\right)$, which are potentially reachable from the seeds by not blocking any vertices are shown in Table 1. Additionally, we discuss a few edge cases by looking at slight variations of BFS as well as SEC with the synthetic graphs. Due to their scale it was computationally infeasible for us given our cluster setup to analyze these on real-world graphs as well. More details on the crawling strategies as well as our seed selection are given in "More details on crawling strategies and seed selection" section.

\section{Evaluation strategy}

The objective of this evaluation is to assess ranking deviations as quantified by Kendall's Tau (cf. "Preliminaries and problem" section) for rankings induced by PAGERANK, computed on the target graph vs. a crawl and compare it against our HAK measure, which is designed to yield values on the same scale. For this, we focus only on high-ranked vertices, as these are typically more interesting in most practical scenarios (Ng et al. 2001a): Firstly, because there is no tangible score difference between the PAGERANK values of the tail vertices. Secondly, ranking deviations in authoritative vertices are typically considered more severe than among the tail ones. Since Kendall's Tau makes no distinctions where rank reversals take place, we compared the ordering among the top 30\%, top 50\% and top 70\% vertices of the crawl and target graph that appeared in both graphs according to the corresponding PAGERANK values. This also helps us characterize where the rank reversals indeed do appear.

The rankings for each of the graphs are computed based on the PAGERANK values. While we employed the regular version PAGERANK on the crawl (with added ghost vertices as sinks), we used the personalized variant of PAGERANK for running it on the target graph. In this version, the algorithm is personalized to a set of vertices, which constitute the starting points as well as teleportation destinations in the algorithm (Page et al. 1999). The resulting PAGERANK values can be interpreted as their importance with respect to these vertices or the domain represented by the crawl. Both variants of PAGERANK ran for 30 iterations with the damping factor parameter set to the frequently cited value of 0.85 .

For a given synthetic (target) graph and a crawling strategy, we repeat each experiment by building different crawls from 100 random seed-sets and provide the average results as well as the $95 \%$ confidence intervals. For real-world graphs we perform experiments by generating crawls from the most important vertices chosen as seeds ( also see "Seed selection" section). We, therefore, run each experiment for real-world graphs corresponding to the crawl generated using this most realistic seed-selection strategy (as against random seed sets employed in synthetic graphs for designing multiple runs) and BFS crawling strategy. 


\section{Crawls and ranking deviations in graphs}

In this section, we aim to answer RQ I and justify the need for estimating ranking deviations before employing PAGERANK for incomplete graphs. In particular, we argue about the results where we witness noticeable ranking deviations of partial crawls with respect to target graphs.

We clearly observe that all real-world graphs exhibit a decreasing $\tau$ with increasing block fraction (see Fig. 3). Most acutely, $\tau$ decreases to 0.55 for the GOV. Synthetic graphs like $G_{n, p}$ and FFBacklinks (first and last row in Fig. 4) exhibit a similar trend with $\tau$ decreasing for increasing block fraction. On the other hand, for the ScaleFree (second row) and ForeFire graphs (third row), we do not witness much change in the ranking orderings, except in the BFS crawls.

A detailed study of the crawls reveals the reasons for such disparate trends for ScaleFree and ForeFire: the crawling strategy combined with the underlying structural properties of the graph sometimes lead to extremely small crawls $(n<1,000)$, much below the desired fraction (cp. "Seed selection and crawling" section). First, we observe a scarcity of backlinks in ForestFire and ScaleFree. That leads to these graphs to be DAG-like without an inadequate number of cycles in the corresponding graphs (cp. "Preliminaries and problem" section). PAGERANK computations over such graphs (or over their subgraphs) tend to finish quickly since the lack of cycles prohibit the random walk to re-cycle back into the graph. This results in small high-fidelity crawls that do not exhibit large ranking deviations when highly linked vertices are prioritized, explicitly (SEC) or by chance (BfsRnd and BfsGeo). Only the BFS strategy that explicitly blocks random vertices causes a deviation in these crawls, as top vertices may be missed as well (conceivable on the Web for different reasons, e.g., restrictive policies and random failures).

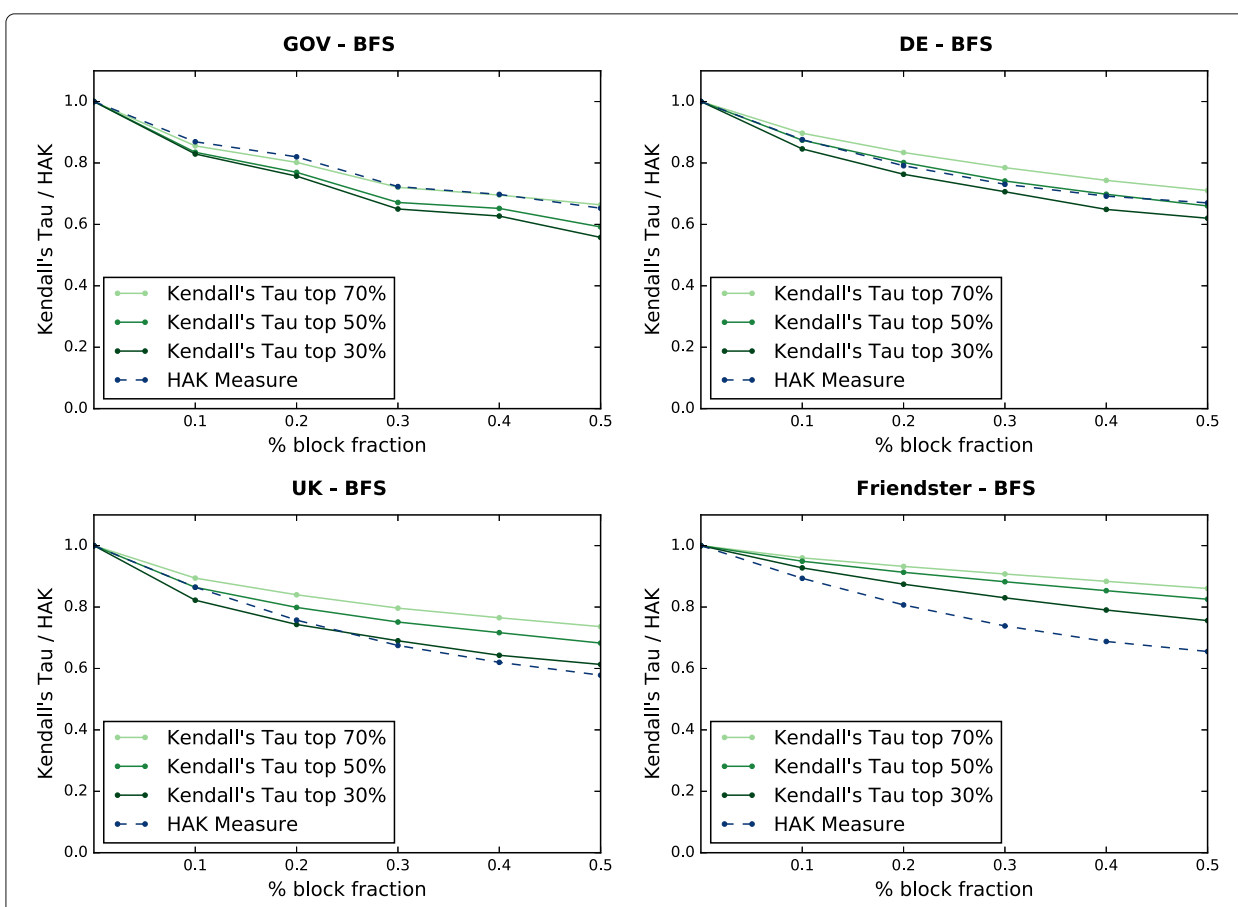

Fig. 3 Ranking deviations measured and estimated for real-world graphs and crawls for different fractions of uncrawled vertices 


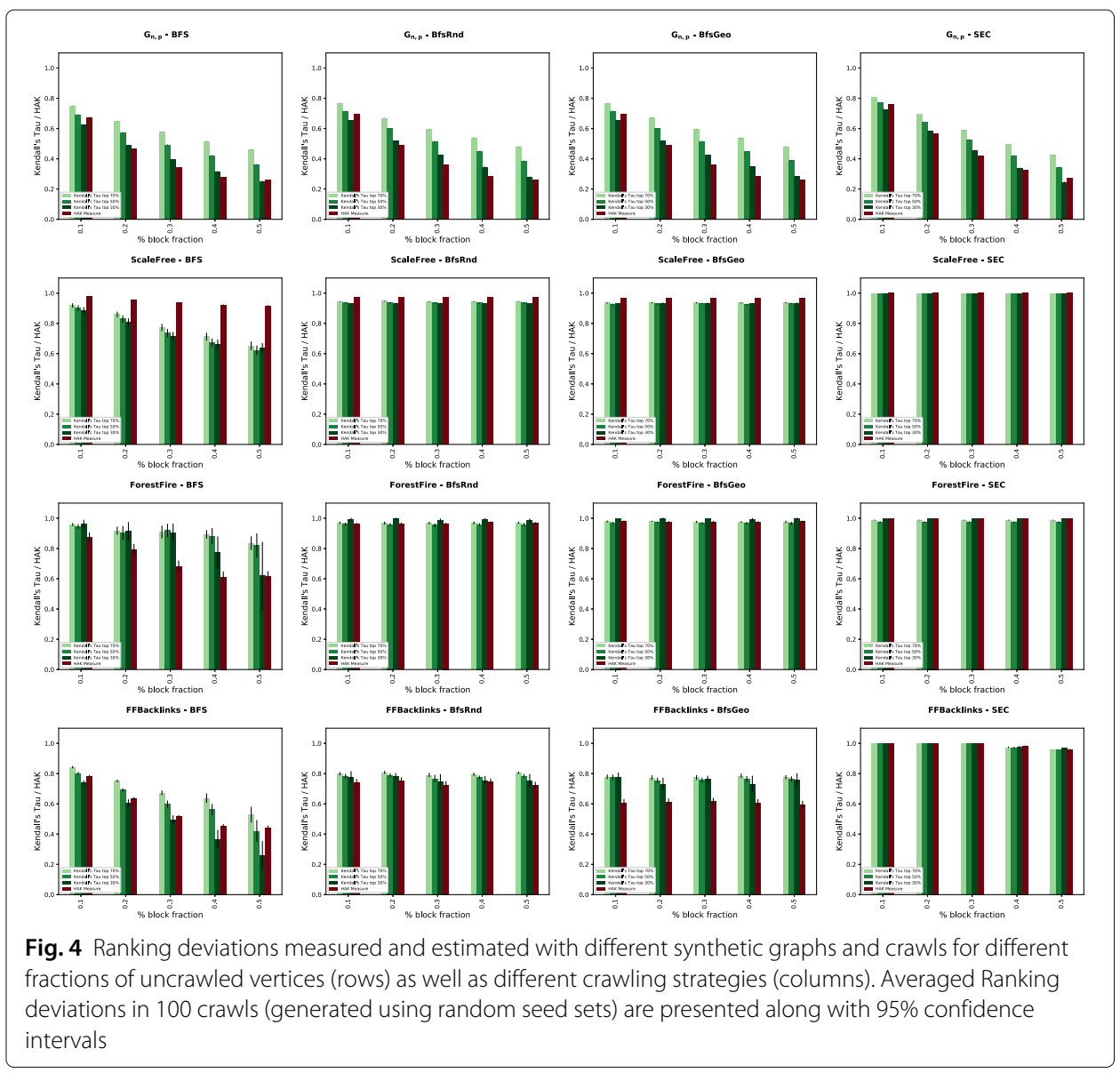

Reinforcing our claim, the addition of backlinks in FFBacklinks resulted in a growing ranking deviation with increasing block fraction with the BFS crawling strategy. We argue that most of the real-world graphs will not be DAG-like and will have backlinks inducing large cycles. Moreover, the random walk nature of PAGERANK computation increases the importance of these backlinks (or feedback loops) towards reaching an equilibrium state. As the core structure of FFBacklinks still resembles the original ForestFire graph, the observed rank deviation is much less severe as compared to $G_{n, p}$.

In addition, we observe that the ranking deviations (in most of the presented cases) increase when we consider a small fraction of the most important vertices. This indicates that most of the low rank vertices in the target graph do not flip their ranks with the more important ones in the crawl, leading to a lower ratio of discordant pairs to the overall total number of pairs. On the other hand, crucial to most applications are the ranking deviations of the high PAGERANK vertices, thus making it essential to monitor them.

Finally, we observe that ranking deviation in the Web graphs shown in Fig. 3 are interestingly similar to the random graphs in Fig. 4 and less so with other generative models like ForestFire or ScaleFree graphs. This, we believe, has strong implications in explaining the structure of Web graphs.

\section{Effectiveness of HAK}

We first discuss about the general applicability of the HAK measure and then argue about the supporting experimental evidence reported in Figs. 3 and 4. We recall that the main 
assumption behind the construction of HAK is that each of the unseen or ghost vertices from the target graph would exert the same fraction of impact (on average) to the crawled set as the actual vertices in the crawl (cp. "The HAK measure" section). We ensure this by constructing the target graph such that each of its vertex has the same fraction of crawled neighbors as the crawled vertices (computed by fidelity). This assumption would not be followed by target graphs, which for example are DAG-like, because the ghost vertices there might not have edges back into the crawl. We remark that HAK cannot identify structures in target graph which are not similar to the crawl, yet leading to severe ranking changes in the crawl. For instance, consider a very small crawl with a very high fidelity and low impact. In such a case HAK would always estimate a very low ranking deviation. It could in the worst happen that there exist a few ghost vertices in the target graph with very high PAGERANK, having outgoing edges to only the low rank vertices in the crawl. Our results in Figs. 3 and 4, on the other hand, support the effectiveness of HAK in most of the studied graphs and therefore also validate our assumptions behind HAK.

We first discuss our findings on synthetic graphs. HAK performs fairly well for $G_{n, p}$, for instance with the BFS crawl strategy with 50\% block fraction, we record an absolute error of 0.007 (actual average: 0.252, estimated: 0.259) for rank correlation of top 30\% vertices. The little ranking deviations in ScaleFree and ForestFire can be attributed to the small crawls with high fidelity $(\gamma \in[0.93,1.0])$. As already discussed, HAK in these cases would always result in a high value, which also explains HAK adapting to the trends. However, we observe a larger deviation for BFS crawls in ScaleFree graphs. Here, HAK underestimates the ranking deviation, which might reflect the existence of the worst case (caused by the random vertex removal in BFS, cf. "Seed selection and crawling" section), resulting in a similar estimation as the one described above for very small crawls. However, HAK overestimates the deviation in FFBacklink (see the middle 2 plots shown in Fig. 4). We attribute this to the fact that the average impact of the crawl increases in presence of backlinks (cp. "Preliminaries and problem" section), which is an overestimation of the actual impact since Forest Fire is nevertheless the dominant topology in this graph. For our measure, a higher average impact corresponds to higher impact on the crawl from the ghost vertices (in our constructed target graph, cp. “The HAK measure” section), leading to increased number of discordant pairs. The uniformly random blocking strategy in the BFS crawls on the other hand might break such backlinks, which lead to a more realistic ranking deviation.

We report more promising results in case of real-world graphs (s. Fig. 3). For instance, for the UK graph we report an almost precise estimation (actual: 0.58, estimated: 0.61). The observed trend in UK is more similar to that seen in to $G_{n, p}$ and FFBacklinks, which might also suggest existence of more backlinks in this graph, leading to large cycles (cp. Fig. 2). In contrast, the deviation in Friendster is less strong and slightly overestimated by HAK (actual: 0.76, estimated: 0.66) similar to ForestFire. We remark here that ForestFire also aims to model social networks and we believe that the similarity of these trends might be caused by the scarcity of backlinks in these graphs. We also note that our estimates reflect more closely the ranking deviations among the top PAGERANK vertices (in either of the compared rankings, cp. "Evaluation strategy" section), which we believe to be more interesting for most practical purposes than deviations in less important vertices or the entire graph. 
Table 3 High-fidelity Component Selection for three largest components (out of 8998 identified components) of the $50 \%$ GOV crawl (with $\boldsymbol{\tau}=\mathbf{0 . 5}$ )

\begin{tabular}{lllll}
\hline & \#Vertices & \#Edges & Fidelity & Kendall's Tau (top 1000) \\
\hline 1. & 12,965 & 17,559 & 0.9822 & 0.9156 \\
2. & 8,836 & 134,582 & 0.6177 & 0.7842 \\
3. & 8,365 & 32,268 & 0.9941 & 0.9130 \\
\hline
\end{tabular}

In summary, the effectiveness of HAK is dependent on the fact that how well we estimate the target graph using properties like fidelity. Moreover, we would require big enough crawls to allow for a representative estimation ${ }^{3}$ of the target graph. We believe that a more sophisticated use of the fidelity and impact of vertices in crawls, for instance using their distributions instead of simple average, will allow us to estimate the target graph and hence the ranking deviation more accurately. In addition, we plan to investigate more properties of the crawled graph, which can be used to predict the corresponding target graph. As our final goal, we will like to extend HAK as a black-box of measures, from which a suitable measure can be chosen in order to estimate ranking deviations in some particular crawl.

\section{High-fidelity component selection}

In this section we discuss the applicability of our proposed algorithm in selecting highfidelity connected subgraphs that have low rank deviation when compared against the target graph. As mentioned in "High-fidelity component selection" section, we provide a heuristic to find high-fidelity components, which might also be of interest for other applications (Borradaile et al. 2012). By applying our algorithm with threshold $\tau=0.5$ to the highly distorted $50 \%$ crawl of the GOV graph (Kendall's Tau of 0.55 , s. "Crawls and ranking deviations in graphs" section), we identified 8998 such components, featuring a reasonably high rank correlation. Table 3 shows the measured values for the three largest of these components, with vertex set sizes ranging from 8,000 to 13,000. As before, the evaluated rankings are based on PAGERANK personalized to the respective component (cp. "Evaluation strategy" section), resulting in correlations measured by Kendall's Tau of over 0.9 . We remark that, since the returned components are very small, a relatively large fraction of vertices with low importance are already present among the top 30\% as studied before (cp. "Evaluation strategy" section). In addition, their interPAGERANK distances are so small, that a small change in their PAGERANK values give rise to a larger number of discordant pairs, resulting in low rank correlation values for the whole component. We, therefore, report the measured values only for the top 1000 vertices.

As our algorithm returns a high number of components and we believe that components with higher fidelity will have correspondingly high rank correlation values, the desired components may be filtered with respect to their size and final fidelity values.

We recall that we optimize for high fidelity instead of our HAK measure with this approach, because of the associated high computational costs. Moreover, due to the unsuitability of using HAK for evaluating small crawls as discussed in "Effectiveness of HAK" section, we do not compare the rank deviations of the identified

${ }^{3}$ We do not want to give recommendations for a minimal size as this is dependent on the target graph and requires some knowledge about it, which should anyway exist when working with a crawl. 
components with their corresponding HAK values. As a matter of fact, for such small components, HAK would almost always return a very high value.

Finally, we conclude two interesting facts from our results: First, our original assumption in HAK about the close relation of fidelity with the rank deviation is justified by our results. We observe that components with higher fidelity also exhibit a higher rank correlation with respect to the target graph. Second, it suffices to use a low threshold $\tau$ to be able to select high-fidelity components. Setting $\tau$ to a higher value increases the fidelity of the returned components, but these components would be too small to be of any practical use.

\section{More details on crawling strategies and seed selection}

\section{Crawling strategies}

- BFS : The breadth-first search (BFS) starts from a set of seed vertices and runs until all vertices are reached. A number of vertices according to the block fraction were chosen uniformly at random and blocked/discarded before the BFS, simulating vertices that cannot be crawled, e.g., due to robots.txt, slow response times, etc.

- BfsRnd : Instead of blocking vertices from the beginning we determine a number of vertices to pursue at each vertex, chosen uniformly at random from its outgoing edges. Additionally, we remove a random number of vertices according to the block fraction from the seed set and run the BFS until the specified desired fraction is discovered.

- BfsGeo : Similar to BfsRnd, but the number of edges to follow was geometrically distributed with parameter $p=0.3$, resembling Forrest Fire Sampling (Leskovec and Faloutsos 2006).

- SEC : In this Sample Edge Count stategy (Maiya and Berger-Wolf 2011), at each step the number of edges from the crawled vertices to all remaining vertices are tracked and those with incoming edges are prioritized.

\section{Seed selection}

We found out that the most realistic seed selection strategy is to pick the most important vertices as seeds. This is also the case for real crawls as these correspond to more wellknown pages. To identify such pages in our target graphs, we first ran PAGERANK on them and constructed the seed set from the top $1 \%$. This allowed us to reduce the size of the large real-world graphs by pre-computing the actual target graphs, consisting only of vertices that are reachable from the seeds (s. Table $1, V_{\text {target }}$ and $E_{\text {target }}$ ). Interestingly, for the GOV and DE graphs, the size difference of the target graphs compared to the originally provided graphs is huge, which confirms common characteristics of these Web archive graphs, i.e., they are not constructed in one crawl, leading to a fairly large number of unimportant vertices (with no in-edges) that were discovered from crawls outside target graphs. The UK and Friendster graphs on the other hand remained at almost the same size, suggesting that they have already been created that way in the first place, which proves our seed selection strategy actually realistic.

\section{Conclusion}

In this paper, we focused on the problem of PAGERANK deviations in Web graphs, typically caused by restricted crawling. We established that deviations in ranking indeed do occur and can be drastic, as shown in our GOV graph where the correlation among the rankings is only 0.55 , measured by Kendall's Tau. To this effect, we proposed the HAK 
measure, which can reliably estimate such deviations purely on the crawl without any knowledge of the original graph. We also presented an algorithm that is capable of selecting multiple connected subgraphs (high-fidelity components) from the crawl in which the relative ordering is much closer to the original ranking.

Our results suggest that crawled Web graphs behave surprisingly similar to random graph models and unlike other generative Web models, such as Forest Fire, in terms of PAGERANK deviations. Thus, this study on crawled Web graphs could be important in studying the structure of the Web as well. For future work, it would be interesting to check if Web graphs are indeed composed of local random structures. Further, from the insights on the effect of backlinks in this work, we intend to look into other representative formal Web models. Also, we would like to investigate the applicability of our measure to determine the confidence of results produced by other centrality measures on partially discovered graphs. Finally, understanding the high-fidelity components might also allow us to identify vertices that cause a severe ranking deviation if not present in a crawl, which is valuable in improving crawling strategies.

Abbreviations

HAK : Holzmann Anand Khosla

\section{Acknowledgements}

We thank the anonymous reviewers for their valuable comments which helped in improving the manuscript. The publication of this article was funded by the Open Access Fund of the Leibniz Universität Hannover.

\section{Authors' contributions}

$\mathrm{HH}$ performed the study and specifically proposed the HAK measure. MK developed the theoretical framework for explaining and supporting the HAK measure. AA proposed experiments to evaluate HAK. All authors analyzed the results and wrote the manuscript.

\section{Funding}

This work is partially funded by SoBigData (European Union's Horizon 2020 research and innovation programme under grant agreement No. 654024).

\section{Availability of data and materials}

This GOV graph is based on crawled webpages provided by the Internet Archive (The Internet Archive 1996). It was extracted from the latest captures of all their archived webpages under the .gov top-level domain (TLD) from 2005 to 2013. The DE graph was also extracted from webpages archived by the Internet Archive, crawled in 2012 and generously provided to us in the project Alexandria ${ }^{4}$. This UK graph TLD crawl from 2005 is publicly available, already in the form of a graph without corresponding webpages (Boldi and Vigna 2004; Boldi et al. 2011). Friendster is a publicly available social network, extracted from an extensive crawl of the former online platform Friendster.com in June 2011 (Archiveteam 2011 ).

\section{Competing interests}

The authors declare that they have no competing interests.

\section{Author details}

${ }^{1}$ Internet Archive, 300 Funston Avenue, San Francisco, USA. ${ }^{2}$ L3S Research Center, Leibniz University, Appelstrasse 9a, 30167 Hannover, Germany.

Received: 27 February 2019 Accepted: 30 August 2019

Published online: 22 October 2019

\section{References}

Archiveteam (2011) Friendster Social Network Dataset: Friends. https://archive.org/details/friendster-dataset-201107, published under CCO 1.0 Universal

Bianchini M, Gori M, Scarselli F (2005) Inside pagerank. ACM Trans Internet Technol (TOIT) 5(1):92-128

Boldi P, Lonati V, Santini M, Vigna S (2006) Graph fibrations, graph isomorphism, and pagerank. RAIRO-Theor Inform Appl 40(2):227-253

Boldi P, Rosa M, Santini M, Vigna S (2011) Layered label propagation: A multiresolution coordinate-free ordering for compressing social networks. In: Srinivasan S, Ramamritham K, Kumar A, Ravindra MP, Bertino E, Kumar R (eds). Proceedings of the 20th international conference on World Wide Web. ACM Press. pp 587-596. https://doi.org/10. $1145 / 1963405.1963488$ 
Boldi P, Santini M, Vigna S (2004) Do your worst to make the best: Paradoxical effects in pagerank incremental computations. In: International Workshop on Algorithms and Models for the Web-Graph. Springer. pp 168-180. https://doi.org/10.1007/978-3-540-30216-2_14

Boldi P, Vigna S (2004) The WebGraph framework I: Compression techniques. In: Proc. of the Thirteenth International World Wide Web Conference (WWW 2004). ACM Press, Manhattan. pp 595-601. http://law.di.unimi.it/datasets.php

Bollobás B, Borgs C, Chayes J, Riordan O (2003) Directed Scale-free Graphs. In: Proceedings of the Fourteenth Annual ACM-SIAM Symposium on Discrete Algorithms. Society for Industrial and Applied Mathematics, Philadelphia. pp 132-139. http://dl.acm.org/citation.cfm?id=644108.644133

Borradaile G, Heeringa B, Wilfong G (2012) The knapsack problem with neighbour constraints. J Discret Algoritm 16:224-235. https://doi.org/10.1016/j.jda.2012.04.011

Costa M, Gomes D, Silva MJ (2017) The evolution of web archiving. Int J Digit Libr 18(3):191-205

Dasgupta A, Kumar R, Sarlos T (2014) On estimating the average degree. In: Proceedings of the 23rd international conference on World wide web. ACM. pp 795-806. https://doi.org/10.1145/2566486.2568019

Erdós P, Rényi A (1959) On random graphs. Publ Math Debr 6:290-297

Ghoshal G, Barabási AL (2011) Ranking stability and super-stable nodes in complex networks. Nat Commun 2:394

Gilbert EN (1959) Random graphs. Ann Math Statist 30(4):1141-1144

Gjoka M, Kurant M, Butts CT, Markopoulou A (2011) Practical recommendations on crawling online social networks. IEEE J Sel Areas Commun 29(9):1872-1892

Hagberg AA, Schult DA, Swart PJ (2008) Exploring network structure, dynamics, and function using NetworkX. In: Proceedings of the 7th Python in Science Conference (SciPy2008)

Hardiman SJ, Katzir L (2013) Estimating clustering coefficients and size of social networks via random walk. In: Proceedings of the 22nd international conference on World Wide Web. ACM. pp 539-550. https://doi.org/10.1145/ 2488388.2488436

Haveliwala T (1999) Efficient computation of pagerank. Tech Rep:1999-31

Haveliwala TH (2002) Topic-sensitive pagerank. In: Proceedings of the 11th international conference on World Wide Web. ACM. pp 517-526. https://doi.org/10.1145/511511.511513

Holzmann H, Anand A, Khosla M (2018) Delusive pagerank in incomplete graphs. In: Complex Networks and Their Applications VII. pp 104-117. https://doi.org/10.1007/978-3-030-05411-3_9

Holzmann H, Nejdl W, Anand A (2016) The dawn of today's popular domains: A study of the archived german web over 18 years. In: Digital Libraries (JCDL), 2016 IEEE/ACM Joint Conference on. IEEE. pp 73-82. https://doi.org/10.1145/ 2910896.2910901

Holzmann H, Nejdl W, Anand A (2017) Exploring web archives through temporal anchor texts. In: Proceedings of the 2017 ACM on Web Science Conference. ACM. pp 289-298. https://doi.org/10.1145/3091478.3091500

Hübler C, Kriegel HP, Borgwardt K, Ghahramani Z (2008) Metropolis algorithms for representative subgraph sampling. In: Data Mining, 2008. ICDM'08. Eighth IEEE International Conference on. IEEE. pp 283-292. https://doi.org/10.1109/icdm. 2008.124

Huurdeman HC, Ben-David A, Kamps J, Samar T, de Vries AP (2014) Finding pages on the unarchived web. In: Proceedings of the 14th ACM/IEEE-CS Joint Conference on Digital Libraries. IEEE Press. pp 331-340. https://doi.org/ 10.1109/jcdl.2014.6970188

Katzir L, Liberty E, Somekh O (2011) Estimating sizes of social networks via biased sampling. In: Proceedings of the 20th international conference on World wide web. ACM. pp 597-606. https://doi.org/10.1145/1963405.1963489

Kendall MG (1938) A new measure of rank correlation. Biometrika 30(1/2):81-93

Kleinberg JM (1999) Authoritative sources in a hyperlinked environment. J ACM (JACM) 46(5):604-632

Leskovec J, Faloutsos C (2006) Sampling from large graphs. In: Proceedings of the 12th ACM SIGKDD international conference on Knowledge discovery and data mining. ACM. pp 631-636. https://doi.org/10.1145/1150402.1150479

Leskovec J, Kleinberg J, Faloutsos C (2007) Graph evolution: Densification and shrinking diameters. ACM Trans Knowl Discov Data 1(1). https://doi.org/10.1145/1217299.1217301

Li RH, Yu JX, Qin L, Mao R, Jin T (2015) On random walk based graph sampling. In: Data Engineering (ICDE), 2015 IEEE 31 st International Conference on. IEEE. pp 927-938. https://doi.org/10.1109/icde.2015.7113345

Maiya AS, Berger-Wolf TY (2011) Benefits of Bias: Towards Better Characterization of Network Sampling. In: Proceedings of the 17th ACM SIGKDD International Conference on Knowledge Discovery and Data Mining. ACM, New York. pp 105-113. http://doi.acm.org/10.1145/2020408.2020431, https://doi.org/10.1145/2020408.2020431

$\mathrm{Ng} \mathrm{AY}$, Zheng AX, Jordan MI (2001a) Link analysis, eigenvectors and stability. In: Proceedings of the 17th International Joint Conference on Artificial Intelligence - Volume 2, IJCAI'01. Morgan Kaufmann Publishers Inc., San Francisco. pp 903-910

$\mathrm{Ng}$ AY, Zheng AX, Jordan MI (2001b) Stable algorithms for link analysis. In: Proceedings of the 24th annual international ACM SIGIR conference on Research and development in information retrieval. ACM, New York. pp 258-266

Page L, Brin S, Motwani R, Winograd T (1999) The PageRank Citation Ranking: Bringing Order to the Web. In: Technical Report. Stanford InfoLab. Previous number = SIDL-WP-1999-0120. http://ilpubs.stanford.edu:8090/422/

Smith JA, Moody J (2013) Structural effects of network sampling coverage i: Nodes missing at random. Soc Netw 35(4):652-668

Smith JA, Moody J, Morgan JH (2017) Network sampling coverage ii: the effect of non-random missing data on network measurement. Soc Netw 48:78-99

The Internet Archive (1996) The Internet Archive. http://archive.org

Vattani A, Chakrabarti D, Gurevich M (2011) Preserving Personalized Pagerank in Subgraphs. In: Proceedings of the 28th International Conference on International Conference on Machine Learning. Omnipress, USA. pp 793-800. http://dl. acm.org/citation.cfm?id=3104482.3104582

Wang DJ, Shi X, McFarland DA, Leskovec J (2012) Measurement error in network data: A re-classification. Soc Netw 34(4):396-409 
Wang T, Chen Y, Zhang Z, Sun P, Deng B, Li X (2010) Unbiased sampling in directed social graph. In: ACM SIGCOMM Computer Communication Review, ACM, vol. 40. pp 401-402. https://doi.org/10.1145/1851275.1851231

Xin RS, Gonzalez JE, Franklin MJ, Stoica I (2013) Graphx: A resilient distributed graph system on spark. In: First International Workshop on Graph Data Management Experiences and Systems, GRADES '13. https://doi.org/10.1145/2484425. 2484427

Zhou Z, Zhang N, Gong Z, Das G (2016) Faster random walks by rewiring online social networks on-the-fly. ACM Trans Database Syst (TODS) 40(4):26

\section{Publisher's Note}

Springer Nature remains neutral with regard to jurisdictional claims in published maps and institutional affiliations.

\section{Submit your manuscript to a SpringerOpen ${ }^{\circ}$} journal and benefit from:

- Convenient online submission

Rigorous peer review

Open access: articles freely available online

- High visibility within the field

- Retaining the copyright to your article

Submit your next manuscript at $\gg$ springeropen.com 